PROCEEDINGS OF THE

AMERICAN MATHEMATICAL SOCIETY

Volume 134, Number 9, September 2006, Pages 2607-2611

S 0002-9939(06)08254-2

Article electronically published on February 17, 2006

\title{
COVERING A BANACH SPACE
}

\author{
VLADIMIR P. FONF AND CLEMENTE ZANCO
}

(Communicated by N. Tomczak-Jaegermann)

\begin{abstract}
A well-known theorem by H. Corson states that if a Banach space admits a locally finite covering by bounded closed convex subsets, then it contains no infinite-dimensional reflexive subspace. We strengthen this result proving that if an infinite-dimensional Banach space admits a locally finite covering by bounded $w$-closed subsets, then it is $c_{0}$-saturated, thus answering a question posed by $\mathrm{V}$. Klee concerning locally finite coverings of $l_{1}$ spaces. Moreover, we provide information about massiveness of the set of singular points in $(\mathrm{PC})$ spaces.
\end{abstract}

A family $\tau=\left\{F_{i}\right\}_{i \in I}$ ( $I$ any set of indices) of subsets of a Banach space $X$ is called a covering of $X$ if $X=\bigcup_{i \in I} F_{i}$. A tiling of $X$ is a covering of $X$ whose members are the closure of their non-empty connected interiors, the interiors being pairwise disjoint. A point $x \in X$ is called a singular point for $\tau$ if each neighborhood of $x$ meets infinitely many members of $\tau$. The set of all singular points for $\tau$ will be denoted by $\operatorname{SP}(\tau)$. A covering $\tau$ is said to be locally finite if $\operatorname{SP}(\tau)=\emptyset$.

The lack of local finiteness in "good" infinite-dimensional Banach spaces as soon as the members of a covering enjoy "nice" properties is far from being a pathological phenomenon. In fact a well-known theorem by H. Corson [C] states that

If an infinite-dimensional Banach space $X$ admits a locally finite covering by closed convex and bounded (CCB in the sequel) sets, then $X$ is not reflexive.

Despite an increasing number of papers dealing with singular points for tilings in infinite-dimensional spaces (see [Z] for references), no more than the quoted Corson's result seems to be available in the literature for coverings. The aim of this paper is to strengthen Corson's theorem. Our approach is different from Corson's, arguments being based on [F3, and it enables us to generalize his theorem in two directions. Essentially, we prove that if an infinite-dimensional Banach space $X$ admits a locally finite covering by bounded weakly closed sets, then $X$ is $c_{0^{-}}$ saturated, i.e. every infinite-dimensional subspace of $X$ (here and in what follows we always mean "closed subspace") contains an isomorphic copy of $c_{0}$. We are therefore able to answer a question posed by V. Klee (Question 2.6, $[\mathrm{K}]$ ); see Corollary 6 below. In fact, we also provide information about distribution and massiveness of singular points. Our work is also an attempt to characterize those separable

Received by the editors October 20, 2004 and, in revised form, March 22, 2005.

2000 Mathematics Subject Classification. Primary 46B20; Secondary 54E52.

Key words and phrases. Covering, locally finite covering, space $c_{0}$, (PC) property.

The first author was supported in part by Israel Science Foundation, Grant \#139/03.

The second author was supported in part by the Ministero dell'Università e della Ricerca Scientifica e Tecnologica of Italy. 
Banach spaces admitting locally finite coverings by "reasonably nice" sets. Such a characterization is already known for tiling by CCB sets. In fact the following is proved in [F2]:

A separable Banach space admits a locally finite tiling by CCB sets if and only if it is isomorphically polyhedral (and hence $c_{0}$-saturated; see [F1]).

Recall that a Banach space is called isomorphically polyhedral if it can be renormed in such a way that any finite-dimensional section of the unit ball is a polytope. So the remaining gap between the necessary and sufficient condition in order to get coverings of a separable Banach space $X$ by bounded $w$-closed sets is actually between $X$ is $c_{0}$-saturated (necessary condition) and $X$ is isomorphically polyhedral (sufficient condition). The second one in fact is strictly stronger than the first one (see $[\mathrm{L}]$ ). So we are lead to propose the following

Conjecture. Any separable Banach space which is $c_{0}$-saturated admits a locally finite covering by bounded $w$-closed sets.

Our results on massiveness of the set of singular points are essentially contained in Theorems 2 and 7 below.

Definition 1. Let $\tau=\left\{F_{i}\right\}_{i \in I}$ be a covering of a Banach space $X$. We say that a subset of indices $\sigma \subset I$ is essential if the set $\bigcup_{i \in \sigma} F_{i}$ is an essential part of a $\tau$, i.e. $G_{\sigma}=X \backslash \bigcup_{i \in I \backslash \sigma} F_{i} \neq \emptyset$.

Theorem 2. Assume that a Banach space $X$ is not saturated by $c_{0}$. Let $\tau=\left\{F_{i}\right\}_{i \in I}$ be a covering of $X$ by $w$-closed bounded sets. Then, for any essential finite set $\sigma \subset I$, the set $\operatorname{cl} G_{\sigma} \cap \mathrm{SP}(\tau)$ is $w$-dense in $\operatorname{cl} G_{\sigma}$.

The proof of Theorem 2 is based on the following result

Proposition 3 ([F3], Corollary 3). Let a Banach space $Z$ contain an open bounded subset $G$ which is a $w-G_{\delta}$-set. Then $Z$ is saturated by $c_{0}$.

Proof of Theorem 2, Let $Y \subset X$ be any infinite-dimensional separable subspace of $X$ with $Y \not \supset c_{0}$, and let $e \in G_{\sigma}=X \backslash \bigcup_{i \notin \sigma} F_{i}$. Put $Z=\operatorname{span}\{e, Y\}$. From now on we work in the space $Z$. Clearly, $Z \not \supset c_{0}$, and $G=G_{\sigma} \cap Z=Z \backslash \bigcup_{i \notin \sigma} F_{i} \neq \emptyset$. Let $W$ be a $w$-open neighborhood of $e$. We prove that the set $\operatorname{cl}(W \cap G)$ contains a singular point for $\tau$, and this will be enough to prove the theorem. Assume that 0 is not in $I$ and set $F_{0}=Z \backslash W$, and $\tilde{I}=I \cup\{0\}$. Clearly, any singular point for the covering $\left\{F_{i}\right\}_{i \in \tilde{I}}$ is a singular point for $\tau$. It is also clear that $G \cap W=Z \backslash \bigcup_{i \in \tilde{I} \backslash \sigma} F_{i}$. Assume to the contrary that the $\operatorname{set} \operatorname{cl}(W \cap G)$ does not contain a singular point for the covering $\left\{F_{i}\right\}_{i \in \tilde{I}}$. Let us show that $G \cap W$ is a bounded open (in the norm topology) and $w-G_{\delta}$ set. The boundedness is clear because $G \cap W \subset \bigcup_{i \in \sigma} F_{i}$, the sets $F_{i}$ 's are bounded, and $\sigma$ is finite. To prove that $G \cap W$ is open, take $x \in G \cap W$ and by using our assumption find a ball centered at $x$ that meets finitely many $F_{i}$ 's, $i \in \tilde{I}$. Next, by using that $x \notin \bigcup_{i \in \tilde{I} \backslash \sigma} F_{i}$, we find a smaller ball centered at $x$ that does not meet any $F_{i}$ with $i \notin \sigma$. Clearly, this ball is contained in $G \cap W$, i.e. $G \cap W$ is open. Next we prove that $G \cap W$ is a $w-G_{\delta}$ set. We again use our assumption, this time for points in $\partial(G \cap W)$. For any $x \in \partial(G \cap W)$ find a ball that meets finitely many $F_{i}$ 's. In particular this ball is covered by a finite union of $F_{i}$ 's. Consider the family $\nu$ of all finite unions of sets $A=F_{i} \cap \partial(G \cap W), i \in \tilde{I} \backslash \sigma$. From the consideration above it is clear that $\partial(G \cap W)=\bigcup_{A \in \nu} \operatorname{int} A$. Since $\partial(G \cap W)$ is a separable metric space, it follows by the Lindelöff theorem that there is a 
countable subfamily $\mu \subset \nu$ such that $\partial(G \cap W)=\bigcup_{A \in \mu}$ int $A$. Now it is clear that there is a countable subfamily $\tau_{1} \subset\left\{F_{i}\right\}_{i \in \tilde{I} \backslash \sigma}$ such that $\partial(G \cap W) \subset \bigcup_{D \in \tau_{1}} D$. Next, $Z \backslash \operatorname{cl}(G \cap W)$ being an open subset of a separable Banach space, it can be represented as $Z \backslash \operatorname{cl}(G \cap W)=\bigcup_{k=1}^{\infty} B_{k}$, where the $B_{k}$ 's are closed balls (in $Z$ ). Finally we have

$$
Z \backslash(G \cap W)=\bigcup_{D \in \tau_{1}}(D \cap Z) \cup \bigcup_{k=1}^{\infty} B_{k} .
$$

Therefore $Z \backslash(G \cap W)$ is $w-F_{\sigma}$, and hence $G \cap W$ is a $w-G_{\delta}$ set. By Proposition 3 the space $Z$ must contain $c_{0}$, a contradiction. The proof is complete.

Corollary 4. Assume that a Banach space $X$ is not saturated by $c_{0}$. Let $\tau=$ $\left\{F_{i}\right\}_{i \in I}$ be a covering of $X$ by $w$-closed bounded sets. Then, for any essential set $\sigma \subset I$, the set $\bigcup_{i \in \sigma} F_{i}$ contains a singular point.

Proof. Assume now that $\sigma \subset I$ is an arbitrary essential set, and let $x \in X \backslash$ $\bigcup_{i \in I \backslash \sigma} F_{i}$. Put $\sigma_{1}=\left\{i \in I: x \in F_{i}\right\}$. Clearly, $\sigma_{1}$ is essential. If $\sigma_{1}$ is finite, then we are done by Theorem 2 If $\sigma_{1}$ is infinite, then $x$ itself is a singular point. The proof is complete.

Corollary 5. Assume that a Banach space $X$ is not saturated by $c_{0}$, and that $\tau=\left\{F_{i}\right\}_{i \in I}$ is a covering of $X$ by bounded $w$-closed sets. Then the set $\operatorname{SP}(\tau)$ is weakly dense in $X$.

Remarks. a) Let us say that a covering $\tau$ of a Banach space $X$ is locally bounded at the point $x \in X$ if there exists a neighborhood of $x$ that meets just bounded members of $\tau$. Since, to prove Theorem 2, boundedness is required only for the $F_{i}$ 's with $i \in \sigma$, the following claim is also true.

Let a Banach space $X$ admit a locally finite covering by $w$-closed sets which is locally bounded at some point. Then $X$ is saturated by $c_{0}$.

b) Note that Corson's theorem cannot be strengthened by asking the members of the covering to be closed and star-shaped. In fact it is proved in [FPZ] that any normed space can be tiled in a locally finite way by bounded closed star-shaped sets.

V. Klee in $[\mathrm{K}]$ constructed a surprising tiling of the space $l_{1}(\gamma), \gamma$ being a suitable big cardinal number, by pairwise disjoint translates of the unit ball. Such a tiling is "extremely non-locally finite", in the sense that each boundary point of each tile is a singular point. In view of the Corson theorem, Klee asked whether it would be possible to cover $l_{1}(\gamma), \gamma$ any infinite cardinal number, by balls in a locally finite way $(\mathrm{K}]$, Queston 2.6). The following corollary answers this question in the negative.

Corollary 6. For $\gamma \geq \aleph_{0}$ the space $l_{1}(\gamma)$ does not admit a locally finite covering by bounded $w$-closed sets (e.g. by balls which are in the above-mentioned Klee's question).

A stronger assumption on $X$ will enable us to get a stronger (than just the $w$-density) property of the set $S P(\tau)$ (see Theorem 7 below).

Recall that a Banach space $X$ is said to have the point of continuity property, briefly (PC) property, if for any separable $w$-closed and bounded subset $A \subset X$ the identity mapping $I d:(A, w) \rightarrow(A,\|\cdot\|)$ has a point of continuity (see [EW]). It 
is easily seen that no Banach space containing $c_{0}$ enjoys the $(\mathrm{PC})$ property, while any Banach space with RNP, in particular any reflexive space, does. It is known (see Theorem 3.13, [EW]) that any $w$-closed bounded subset of any (PC) space is a Baire space in the weak topology. If in addition the space is separable, then even any (norm-)closed bounded subset of it, being a $w-G_{\delta}$ set, is a Baire space in the weak topology. It is not difficult to see (by using Proposition 3.9 and Theorem 3.13, $[\mathrm{EW}]$ ) that for any (norm-)closed bounded subset $A \subset X$ of a separable space with (PC) property, the set $C(A)$ of all points in $A$ of weak-to-norm continuity is a $w$-dense and $w-G_{\delta}$ subset of $A$.

Theorem 7. Let $X$ be a separable infinite-dimensional Banach space with (PC) property, and let $\tau=\left\{F_{i}\right\}_{i \in I}$ be a covering of $X$ by w-closed bounded sets. Let $\sigma \subset I$ be a finite essential set and let $G_{\sigma}=X \backslash \bigcup_{i \in I \backslash \sigma} F_{i}$. Then the set $S P(\tau) \cap \operatorname{cl} G_{\sigma}$ is $w$-dense and of the second category in $\mathrm{cl} G_{\sigma}$.

Proof. $w$-density of $S P(\tau) \cap \operatorname{cl} G_{\sigma}$ in $\operatorname{cl} G_{\sigma}$ was already proved in Theorem 2, Note that $S P(\tau) \cap \operatorname{cl} G_{\sigma}=S P(\tau) \cap \partial G_{\sigma}$. Put $S=\operatorname{SP}(\tau) \cap \partial G_{\sigma}$ and assume to the contrary that $S$ is of the first category in $\mathrm{cl}_{\sigma}$, i.e. $S \subset \bigcup_{k=1}^{\infty} D_{k}$, where each $D_{k}$ is $w$-closed and nowhere $w$-dense in $\operatorname{cl} G_{\sigma}$. Note that $G_{\sigma} \cap\left(\partial G_{\sigma} \backslash S\right)=\emptyset$, and any point in the set $\partial G_{\sigma} \backslash S$ is a point of local finiteness. By using the Lindelöff theorem for the set $\partial G_{\sigma} \backslash S$ (as it was done in the proof of Theorem 21), we can find a sequence of sets $\left\{F_{i_{k}}\right\}_{k=1}^{\infty}, i_{k} \in I \backslash \sigma, k=1,2, \ldots$, such that $\partial G_{\sigma} \backslash S \subset \bigcup_{k=1}^{\infty} F_{i_{k}}$. Finally, the set $X \backslash \operatorname{cl} G_{\sigma}$, being an open subset of a separable Banach space, can be represented as $X \backslash \mathrm{cl} G_{\sigma}=\bigcup_{k=1}^{\infty} B_{k}$, where the $B_{k}$ 's are closed balls. Put

$$
K=\bigcup_{k=1}^{\infty}\left(B_{k} \cup F_{i_{k}} \cup D_{k}\right), \quad H=X \backslash K .
$$

Clearly, $H \subset G_{\sigma}$ and $H$ is a weak $G_{\delta}$ set (in $X$ ). We claim that $H=\operatorname{cl} G_{\sigma} \backslash K$ is $w$-dense in $\operatorname{cl} G_{\sigma}$. We have $\operatorname{cl} G_{\sigma} \backslash K=\operatorname{cl} G_{\sigma} \backslash \bigcup_{k=1}^{\infty}\left(F_{i_{k}} \cup D_{k}\right)$, where $F_{i_{k}}$ 's and $D_{k}$ 's are weakly closed and do not contain any $w$-open set in $\mathrm{cl}_{\sigma}$ (recall that $\left.F_{i_{k}} \cap G_{\sigma}=\emptyset\right)$. Assume to the contrary that there is a $w$-open subset $V$ of $\operatorname{cl} G_{\sigma}$ with $V \subset \bigcup_{k=1}^{\infty}\left(F_{i_{k}} \cup D_{k}\right)$. Since $\operatorname{cl} G_{\sigma}$ is a Baire space in the weak topology, so it is $V$. Hence there is an index $k$ such that either $F_{i_{k}}$ or $D_{k}$ contains a $w$-open subset of $\mathrm{cl}_{\sigma}$, a contradiction. Thus we proved that $H$ is $w$-dense in $\operatorname{cl} G_{\sigma}$. Now let $C\left(\mathrm{cl} G_{\sigma}\right)$ be the set of all points in $\mathrm{cl}_{\sigma}$ of weak-to-norm continuity. As it was already mentioned, this set is $w$-dense and $w-G_{\delta}$ in $\mathrm{cl}_{\sigma}$. Since $\mathrm{cl} G_{\sigma}$ is a Baire space, it follows that $H \cap C\left(\operatorname{cl} G_{\sigma}\right)$ is non-empty (and even $w$-dense in $\operatorname{cl} G_{\sigma}$ ). It is not difficult to see that any point in $H \cap C\left(\operatorname{cl} G_{\sigma}\right)$ is a singular point for $\tau$. However, by our construction, $H \cap S P(\tau)=\emptyset$, a contradiction. The proof is complete.

Remark. As the following example shows, the set $G=X \backslash \bigcup_{i \in I \backslash \sigma} F_{i}$ may not contain any singular point (i.e. closing $G_{\sigma}$ in Theorems 2 and 7 is necessary). Call $F_{0}$ the closed unit ball $B_{X}$ of $X$, and let $F_{x}=\{x\}$, for any $x \in X \backslash \operatorname{int} B_{X}$. If $\sigma=\{0\}$, then $G_{\sigma}=\operatorname{int} B_{X}$. Clearly, $G_{\sigma}$ does not contain any singular point.

However, if a covering is countable, a slight modification of the proofs shows that closing $G_{\sigma}$ may be omitted in Theorems 2 and 7. 


\section{ACKNOWLEDGMENTS}

This paper was completed when the first-named author was visiting the Mathematical Department of the Università degli Studi of Milano, Italy. He is grateful for their kind hospitality.

\section{REFERENCES}

[C] H.H. Corson, Collections of convex sets which cover a Banach space, Fund. Math. 49 (1961), 143-145. MR0125430(23:A2732)

[EW] G.A. Edgar and R.F. Wheeler, Topological properties of Banach spaces, Pacific J. Math. 115 (1984), 317-350. MR0765190 (86e:46013)

[F1] V.P. Fonf, Polyhedral Banach spaces, Math. Notes USSR 30 (1981), 809-813. MR0638435 (84j:46018)

[F2] V.P. Fonf, Three characterizations of polyhedral Banach spaces, Ukrainian Math. J. 42 (1990), 1286-1290. MR1093646 (92e:46028)

[F3] V.P. Fonf, Boundedly complete basic sequences, $c_{0}$-subspaces and injections of Banach spaces, Isarel J. Math. 89 (1995), 173-188. MR.1324460 (96a:46021)

[FPZ] V.P. Fonf, A. Pezzotta and C. Zanco, Singular points for tilings of normed spaces, Rocky Mountain J. Math. 30 (2000), 857-868. MR.1797820 (2001k:46020)

[K] V. Klee, Dispersed Chebyshev sets and covering by balls, Math. Ann. 257 (1981), 251-260. MR0634466 (84e:41036)

[L] D.H. Leung, Some isomorphically polyhedral Orlicz sequence spaces, Israel J. Math. 87 (1994), 117-128. MR 1286820 (95f:46033)

[Z] C. Zanco, Even infinite-dimensional Banach spaces can enjoy carpeting and tiling, Proc. of the 13th Seminar on Analysis and its Applications, Isfahan Univ. Press, Isfahan, 2003. MR.2114509

Department of Mathematics, Ben-Gurion University of the Negev, 84105 Beer-Sheva, ISRAEL

E-mail address: fonf@math.bgu.ac.il

Dipartimento di Matematica, Università degli Studi, via C. Saldini 50, 20133 Milano MI, ITALY

E-mail address: zanco@mat.unimi.it 